\title{
Is Fast Track Cardiac Anaesthesia Cost Effective without Compromising Morbidity and Mortality?
}

\author{
Puja Vimesh M.D., ${ }^{1}$ Shyam Singh, M.Ch., ${ }^{2}$ Shivani Mehta, M.D., ${ }^{3}$ Ramesh Chander, M.D. ${ }^{4}$ \\ 'Department of Cardiothoracic Anaesthesiology Super-Speciality Hospital, Govt. Medical College Jammu \\ ${ }^{2}$ Department of Cardiovascular and Thoracic Surgery, Sher-i-Kashmir Institute of Medical Sciences, Soura Srinagar \\ ${ }^{3}$ Department of Pathology, Govt. Medical College, Jammu \\ ${ }^{4}$ Department of Community Medicine, CMC \& H, Bungal, Pathankot, Punjab
}

\section{A B S T R A C T}

Background: With the evolution of anaesthesia and surgical procedures, fast track cardiac anaesthesia (FTCA) has gained an increased interest, mainly based on the possibility of reducing health costs without compromising patient care and wellbeing Objective:The purpose of this study was to evaluate different durations in Early Extubation (EE) like Aortic Cross Clamp Time (ACCT), Cardiopulmonary bypass time (CPB Time), Mechanical Ventilation Duration (MVD), Operation Room time (ORT), Intensive Care Unit (ICU) and post ICU time as well as Total Hospital Stay Time (THST) in open heart procedures. This has been done to co-relate FTCA with lowering of hospital expenditure without compromising with morbidity and mortality.

Methods: This is a "Prospective Cohort Study" comprising of a group of 80 patients who underwent open heart surgery in a Rural Cardiac Center, in 2014. After FTCA and surgical technique, we reviewed the duration of mechanical ventilation, length of Intensive Care Unit stay, reintubation, and incidence of in-hospital mortality and followed our cases prospectively till discharge from the hospital.

Results : The group of Fast Track Cardiac Anaesthesia comprised of 80 patients. Range of ventilation duration (VD) was 2-4 hours in 12.8\%) to $8-10$ hours in 9\% cases; Aortic cross clamp time (ACCT) in minutes was $25-49$ in $35 \%$ cases to $100-124$ in $2.7 \%$ of cases. Similarly Range of Cardio-pulmonary Bypass Time (CPBT) in minutes 40-69 minutes in 21.6\% to 160-190 minutes in $4 \%$ cases, surgery time (ST) in hours was 23 in $7.5 \%$ cases to $6-8$ in $28.8 \%$ cases; operation room time (ORT) in hours was $2-4$ in $3.8 \%$ to $6-8$ hours in $28.8 \%$ patients; ionotropic duration (ID) in hours was $2-5$ in $20 \%$ to 18 to 24 hours in $6.3 \%$ cases; Intensive Care Unit (ICU) stay in days was $1-2$ days in $66.3 \%$ to $3-4$ days in $3.8 \%$ cases ; and post ICU stay in days was $2-3$ in $1.3 \%$ to $6-7$ in $87.5 \%$ patients. Total hospital stay in days was $4-6$ days in 4 case, $7-9$ in 40 cases and 10-12 days in 36 cases. Reintubation was done only in 1 out of 78 cases. In our study there was no mortality. Conclusions :Fast track Cardiac anaesthesia is suitable with applied anesthetic technique in our centre. However there exists relatively small risk of re-intubation (1.3\%). Small sample size is pointer towards a little lack of statistical strength of the study. JMS 2017;20(1):22-30

Keywords: CardiacAnaesthesia, fast-track, re-intubation, CPB time, ACC time, Mechanical ventilation.

\section{INTRODUCTION}

Fast Track Cardiac Anaesthesia (FTCA) is one of the biggest discovery of anaesthesiology. FTCA is Early Extubation Anaesthesia $\{$ EEA $\}$ within 8 hours of the end of surgery. It is based on the administration of relatively small amounts of short-acting opioids, supplemented with either propofol or volatile anesthetics. ${ }^{1}$ It has been reported that the risk of cardiovascular complications after FTCA is comparable with Conventional Cardiac Anaesthesia (CCA). Nonetheless, the safety of FTCA has not been

\section{Correspondence}

Dr. Puja Vimesh

Assistant Professor \& Head

Department of Cardiothoracic Anaesthesiology

Super-Speciality Hospital, Govt. Medical College Jammu E Mail: pujavimesh@rediffmail.com thoroughly evaluated, because the studies performed to date enrolled too few patients to adequately assess the relative risk for low-frequency complications and mortality. ${ }^{2}$

Many major centers, however, aim for extubation 14 hours postoperatively. There is also the notion of UltraFast Track Anaesthesia (UFTA), which refers to extubation of patients in theatre post operatively. It is mostly reserved for patients undergoing off-pump cardiac surgery but some work has been done on patients after cardiopulmonary bypass (CPB) and it is generally not a universally accepted method. ${ }^{3}$. Patients eligible for a fast track protocol should include: Adult patients- Elective surgery- Either CABG / valve surgery- 1 st time surgery- Good LV function- No recent myocardial infarction (MI) i.e. within 30 days of 
surgery- No systemic disease that would contraindicate the fast tracking of the patient e.g. renal failure; chronic obstructive pulmonary disease. ${ }^{4}$ The two largest studies comparing FTCA with CCA, in fact, comprised only 1012 and 404 patients yielding results with limited statistical power. Meta-analyses evaluating FTCA included randomized trials with predominantly low-risk patients and may have been subjected to publication bias and other limitations inherent with this analytic approach. ${ }^{5}$

Haanschoten, M.C., Van Straten, et al 6 did a metaanalysis with their objective being to prove that FTCA was as safe as Conventional Cardiac Anaesthesia (CCA). They included all trials involving elective $\mathrm{CABG}$ or valve surgeries. They concluded that there was no evidence of increased morbidity or mortality with FTCA techniques and combined with the known cost benefits there was no reason to continue with high dose opioid techniques . They did concede, however, that even as a meta-analysis their study was underpowered and therefore unable to accurately predict rare events including mortality. Early extubation also showed a decreased ICU and hospital length of stay. Hantschel D., Fassl J., Scholz M, et al7 published their study in Anesthesia \& Analgesia. Their aim was to compare FTCA and CCA using a large number of patients 7989 surpassing the 2 previous largest studies with 1012 and 4042 patients respectively. They excluded any off-pump cardiac surgery and any patients who received any form of neuraxial blockade.

\section{METHODS}

The present study is a prospective cohort study, comprising of a group of 80 patients of all age groups, of both sexes, who were operated in 2014. This study was conducted in the "Rural Centre for Cardio-Thoracic and Vascular Diseases", Dr. K. M. Cherian Hospital Parumala, district Pathanamthitta, Kerala state, India. We followed the cohort from the date of admission prospectively through surgery day and then post-operative period in the OT, ICU, Post ICU, in the wards till discharge. This way we calculated, ventilation period ( Extubation period), OR time, ACCT, ICU period, Post ICU period, and total hospital stay, besides other periods as shown in different tables and figures.

\section{Anaesthesia Technique:}

All the 80 patients receiving FTCA, premedication consisted of oral diazepam 5 to $10 \mathrm{mg}$, at night. In the operating room monitoring of ECG and pulse oximetry was initiated. Under local anaesthesia and aseptic precautions, a 16-G intravenous cannula was inserted in the cephalic or cubital vein and a 20-G intra-arterial cannula was introduced into the radial artery for invasive beat to beat monitoring of the arterial pressure. Oxygen was administered via nasal prongs at 3 to $4 \mathrm{~L} / \mathrm{min}$ as a protocol in our cardiac theatre. FTCA is based on the administration of relatively small amounts of short-acting opioids, supplemented with either propofol or volatile anesthetics and Anaesthesia was induced with midazolam $0.05 \mathrm{mg} / \mathrm{kg}$, fentanyl 5 to $10 \mathrm{mcg} / \mathrm{kg}$ (low dose opioids), ketamine 0.5 to $1 \mathrm{mg} / \mathrm{kg}$ intravenously. Tracheal intubation was facilitated with vecuronium 0.08 to $0.1 \mathrm{mg} / \mathrm{kg}$. Anaesthesia was maintained with infusion of propofol at the rate of 100 $\mathrm{mcg} / \mathrm{kg} / \mathrm{min}$ through infusion pump and isoflurane. ${ }^{8}$

Ventilation was done by giving oxygen in the concentration of 0.5 to $1 \%, 5-6$ litres on closed circuit on Drager Anaesthesia Machine. Once eye lash reflex was abolished for about 3-4 minutes, intubation was done with Portex ETT of appropriate size. Nasopharyngeal probe was inserted and fixed. Transoesophageal Probe was introduced at mid-oesophageal level so that all the chambers of the heart were visualized on the echo-cardiograpic machine. Air and oxygen combination was initiated in the ratio of 60 to 40 before cardiopulmonary bypass (CPB). Urinary catheter was introduced to monitor urinary output during pre-bypass, on bypass and post bypass period as important indicator of vital perfusion.9 In high risk patients (unstable angina, Left Main Disease, Triple Vessel Disease, Ejection fraction $<40 \%$ ) Swanganz catheter was introduced through sub-clavian approach and pre-operative CO, CI, SVR, PVR, PA pressures, PA wedge pressure were noted. In Low risk patients triple Lumen CVP was introduced. Before initiation of cardiac surgical procedure Heparin in the dose of 3 to $4 \mathrm{mg} / \mathrm{kg}$ was introduced through the Central cannula and after attaining ACT above 480 seconds, surgeons were allowed to go on CPB machine.9After initiation of CPB, propofol infusion was continued and fentanyl 5 to 10 
$\mathrm{mcg} / \mathrm{kg}$ and vecuronium 0.1 to $0.2 \mathrm{mg} / \mathrm{kg}$ were added intermittently through CPB machine (in strict titrated dose). Standard surgical and perfusion techniques were used in all patients. Inspired and expired gas concentration of oxygen $(\mathrm{O} 2)$, carbon dioxide $(\mathrm{CO} 2)$ and isoflurane were measured using anaesthetic gas monitoring system. Haemodynamic parameters were maintained within $20 \%$ of the basal values with small boluses of IV nitroglycerine or ephedrine / phenylephrine / noradrenaline and small boluses of IV metoprolol / esmolol or atropine / epicardial pacing as required. Serial ABG's and ACT were done half hourly. Once the surgical procedure was over, heparin was neutralized with protamine as an anti-dote, venous cannulae were removed, followed by the removal of the aortic cannula. Filling pressures and fluid balance was maintained using lactated Ringers solution, Gelofusine, blood and blood products as necessary. If needed, infusions of dopamine / milrinone / adrenaline / noradrenalin / nitroglycerin were used as inotropes / vasodilators while coming off by-pass to maintain haemodynamics. ${ }^{10}$

At the end of surgical procedure, patients were shifted to surgical intensive care unit (SICU). Propofol infusion was maintained at the rate of $25-50 \mathrm{mcg} / \mathrm{kg} / \mathrm{min}$ for one to four hours in the SICU. Analgesia in the SICU was provided by low dose intravenous Morphine. Patients were observed for stable vital parameters and fulfilling the extubation criteria. Patient was warmed with forced air warming system (Bair Hugger Warming Unit, Arizant Healthcare Inc. MN, USA) if required. All the patients were ventilated in synchronized intermittent mandatory mode of ventilation (SIMV) with pressure support. These ventilated patients were weaned and then extubated by standard protocol when haemodynamically stable awake, without signs of residual neuromuscular blockade. Mechanical ventilation was continued with sedation if patient had excessive drains or advised by attending physician. One of our patient had excessive drains and he was not fit for Fast Track Anaesthesia and put on Conventional Anaesthesia and so extubation was withheld till the patient was haemodynamically stable and so this particular patient was excluded from our study. Duration of mechanical ventilation was defined as time from skin closure to extubation of trachea.

\section{Data Collection}

Clinical data for the patients were prospectively registered in a dedicated electronic research database from admission until hospital discharge. In the operating room, the attending anesthesiologist documented the patients' demographic data and intraoperative data on a dedicated form and also entered these variables in an electronic database (Microsoft Access). These data included Age distribution, sex distribution, Aortic Cross clamp time (ACCT) in minutes, Cardio- pulmonary bypass time (CPB time) in minutes, surgery time (ST) in hours, Total Operation time OT) in hours, Ionotrope duration Time (IDT) in hours, duration of mechanical Ventilation (VDT) in hours. After the patients were admitted in the ICU, relevant data (duration of ventilation, length of ICU stay, medication, complications, and routine laboratory measurements) were collected by the ICU medical staff on another dedicated data form. After transfer to the ward, additional data (length of hospital stay, medication, and complications) were recorded by nurses from the research team. These research nurses subsequently entered the data from the dedicated forms into the electronic research database. Because the demographic and intra-operative data were already entered into the database by the attending anesthesiologist, the nurses did not re-enter these data but only compared these electronic and written variables. After the patient's discharge from the hospital, all data collection forms were rechecked by another member of the research team to confirm accuracy of the data entry. The final data were transferred into an SPSS database version 20, from which further analyses could be performed.

\section{RESULTS}

The total sample size was 80. Age distribution range was from $<30$ years to 80 years and frequency is depicted in table 1.Male and female ratio of patients was 66 : $14(82.5 \%: 17.5 \%)$. Cases operated under FTCA were: Coronary artery disease(CAD) with Tripple vessel disease (TVD) : 65,CAD with double vessel disease (DVD): 5,Atrial septal defect (ASD): 6, Aortic stenosis(AS): 1,Mitral regurgitation (MR): 2,Aortic regurgitation(AR): 1. Surgeries done : CABG on pump 64, ASD primary repair 4, ASD patch closure 2, CABG off pump 4, MVR 2, AVR 2, 
CABG off pump(Awake) 2, (Table 1).

Cardio- pulmonary bypass time in minutes was: 40-69 minutes : 16(21.6\%), 70-99 minutes: $15(20.3 \%)$, 100-129 minutes: $28(37.8 \%)$, 130-159 minutes: $12(16.2 \%)$ and 160-190 minutes : 3 cases(4.05); 6 cases of CABG were operated off pump. Aortic CCT in minutes in FTA study subjects was: $25-49$ minutes: 26 cases (35.1\%), 50-74 minutes 35 cases (47.3\%), 75-99 minutes 11 cases (14.86\%), 100-124 minutes 2 cases $(2.7 \%)$; 6 cases of CABG were performed off pump. Total surgery time in hours was: 2-3 hours : 6 cases, 3-4 hours : 26, 4-5 hours : 31 , 5-6 hours : 14 and 6-7 hours : 3 cases respectively. (Figure1). Total Operation room time (OR) was 2-4 hours: 3 cases, 4-6 hours: 54 and 6-8 hours: 23 cases). Ionotropic duration time was: 2-5 hours 16 cases, 6-9 hours 17, 10-13 hours $16,14-17$ hours 18 and 18 to 24 hours were 13 cases respectively. Ventilation duration time (extubation time) was: 2-4 hours: 10 cases (12.8\%), 4-6 hours 21 (26.9\%), 6-8 hours $40(51.3 \%)$, and $8-10$ hours only 7 out of 78 cases respectively $(9 \%) ; 2$ cases underwent awake CABG. Reintubation was done only 1 out of 78 cases in our cardiac centre .ICU stay in days was 1-2 days 53 cases, 2-3 days 24 cases and 3-4 days 3 days respectively. Post ICU stay was 23 days 1 case, 4-5 days 9 cases, 6-7 days 70 cases respectively. Total hospital stay in days was 4-6 days in 4 case, 7-9 in 40 cases and 10-12 days in 36 cases. (Table2) There was no mortality in this study.

\section{DISCUSSION}

The study under discussion comprised of 80 open heart surgery cases, Our cardiac center has embraced FTCA protocols and the objective of the study was to assess its cost effectiveness by reducing extubation time, OR duration, ICU and post ICU bed use duration and total hospital stay. Mechanical Ventilation duration in the present FTCA group was 2-4 hours in 10 cases (12.8\%), 4-6 hours 21 cases $(26.9 \%), 6-8$ hours in 40 cases $(51.3 \%)$, and $8-10$ hours only in 7 cases (9\%) (Table 2). Herein 91\% were extubated in less than 8 hours and out of which $39.7 \%$ in less than 6 hours. In comparison it is less than half of conventional cardiac anaesthesia (CCA) cases (12 hours) (36) In a study by Hemmerling TM (11) The duration of mechanical ventilation was shorter in the FTCA patients compared with the CCA group (6 vs $12 \mathrm{~h}, \mathrm{P} \leq 0.001)$, Conclusively this was related to low morbidity and low cost of hospital expenditure12. The expenditure of heart surgeries is one of the highest anywhere and everywhere, as such it's reduction is a great boon to the cardiac surgery patients.(13) Another research by Silbert BS(14) expressed different view which was not in agreement with us : that the duration of mechanical ventilation was shorter in the patients receiving FTCA, but this did not result in a shorter ICU stay. Patients in the FTCA group were discharged from the hospital later than CCA patients. We are lacking in valid explanation for this finding. May be the higher rates of co morbidity in the former group required more postoperative care, or that there was a higher rate of other complications.

Aortic CCT in minutes in FTA study subjects was: 25-49 minutes: 26 cases (35.1\%), 50-74 minutes: 35 cases (47.3\%), 75-99 minutes 11 cases (14.86\%) and 100-124 minutes 2 cases $(2.7 \%)$; 6 cases underwent off pump CABG.

Our results are in agreement with studies conducted by Jeju Nath Pokharel, Apurba Sharma et al in National Heart Centre, Bansbari, Kathmandu, Nepal (NHJ Volume 10 Number 1 Issue 1 November 2013) and many others $(15,16)$ In the present study Cardio- pulmonary bypass time in minutes was: 40-69 minutes in16 (21.6\%), 70-99 minutes in15 (20.3\%), 100-129 minutes in 28(37.8\%), 130-159 minutes in12 (16.2\%) and 160-190 minutes in 3 cases $(4.05 \%)$. ( Table 2$)$. The study conducted by Siliciano D etal17 showed results which are in agreement with our results. Total surgery time in hours was: 2-3 hours 6 cases, 3-4 hours 26, 4-5 hours 31, 5-6 hours 14 and 6-7 hours 3 cases respectively (Figure 1). Rady MYetal18 agree with us in their study results. The research conclusions of Total Operation room time (OR) was 2-4 hours 3 cases, 4-6 hours 54 cases, 6-8 hours 23 cases. (Table 2). Michalopoulos A. et al 19 in their study differ with us on the point that total operation time is not significantly different from CCA if critical statistical analysis is done. Ionotropic duration was: 2-5 hours 16 cases, 6-9 hours 17 cases, 10-13 hours 16 cases, and 14-17 hours 18 cases and 18 to 24 hours were in 13 cases respectively (Table 2 ). However, results of study by Kataoka G 20 are different than our results. Over all 
durations in our FTCA study were significantly lower than traditional anaesthesia. In a study conducted by Ender J 21 Duration of anaesthesia, min (mean + SD) $134.8 \pm 35.2$. In our study only one case was reintubated overall (incidence
1.3\%) Table 2. A study was conducted by LaheySJetal22 showed overall five of 277 (1.8\%) patients had to be reintubated.

TABLE1: AGE \& SEX WISE DISTRIBUTION; CASES OPERATED AND TYPES OF SURGERIES PERFORMED IN THE STUDY SUBJECTS UNDER FTCA.

\begin{tabular}{|c|c|c|c|c|}
\hline \multicolumn{5}{|c|}{ AGE DISTRIBUTION: } \\
\hline & Frequency & Percent & $\begin{array}{c}\text { Valid } \\
\text { Percent }\end{array}$ & $\begin{array}{c}\text { Cumulative } \\
\text { Percent }\end{array}$ \\
\hline$<30$ years & 3 & 3.8 & 3.8 & 3.8 \\
\hline $30-39$ & 5 & 6.3 & 6.3 & 10.0 \\
\hline $40-49$ & 17 & 21.3 & 21.3 & 31.3 \\
\hline $50-59$ & 24 & 30.0 & 30.0 & 61.3 \\
\hline $60-69$ & 26 & 32.5 & 32.5 & 93.8 \\
\hline $70-80$ & 5 & 6.3 & 6.3 & 100.0 \\
\hline \multicolumn{5}{|c|}{ SEX DISTRIBUTION } \\
\hline Male & 66 & 82.5 & 82.5 & 82.5 \\
\hline Female & 14 & 17.5 & 17.5 & 100.0 \\
\hline Total & 80 & 100.0 & 100.0 & \\
\hline \multicolumn{5}{|c|}{ CASES OPERATED } \\
\hline CAD with TVD & 65 & 81.3 & 81.3 & 81.3 \\
\hline CAD with DVD & 5 & 6.3 & 6.3 & 87.5 \\
\hline ASD & 6 & 7.5 & 7.5 & 95.0 \\
\hline AS & 1 & 1.3 & 1.3 & 96.3 \\
\hline MR & 2 & 2.5 & 2.5 & 98.8 \\
\hline AR & 1 & 1.3 & 1.3 & 100.0 \\
\hline Total & 80 & 100.0 & 100.0 & \\
\hline \multicolumn{5}{|c|}{ SURGERIES DONE } \\
\hline CABG on pump & 64 & 80.0 & 80.0 & 80.0 \\
\hline ASD Pry. Repair & 4 & 5.0 & 5.0 & 85.0 \\
\hline ASD Patch closure & 2 & 2.5 & 2.5 & 87.5 \\
\hline CABG off pump & 4 & 5.0 & 5.0 & 92.5 \\
\hline MVR & 2 & 2.5 & 2.5 & 95.0 \\
\hline AVR & 2 & 2.5 & 2.5 & 97.5 \\
\hline $\begin{array}{l}\text { CABG off pump } \\
\text { (Awake) }\end{array}$ & 2 & 2.5 & 2.5 & 100.0 \\
\hline Total & 80 & 100.0 & 100.0 & \\
\hline
\end{tabular}


TABLE 2: DIFFERENT DURATIONS UNDER FAST TRACT CARDIAC ANAESTHESIA IN STUDY SUBJECTS

\begin{tabular}{|c|c|c|c|c|}
\hline \multicolumn{5}{|c|}{ AORTIC CROSS CLAMP TIME IN MINUTES } \\
\hline $25-49$ & 26 & 35.1 & 35.1 & 35.1 \\
\hline $50-74$ & 35 & 47.3 & 47.3 & 82.4 \\
\hline $75-99$ & 11 & 14.9 & 14.9 & 97.3 \\
\hline $100-124$ & 2 & 2.7 & 2.7 & 100 \\
\hline Total & 74 & 100 & 100 & 100 \\
\hline \multicolumn{5}{|c|}{ CPB TIME IN MINUTES IN STUDY SUBJECTS } \\
\hline 40-69 & 16 & 21.6 & 21.6 & 21.6 \\
\hline $70-99$ & 15 & 20.3 & 20.3 & 41.9 \\
\hline $100-129$ & 28 & 37.8 & 37.8 & 79.7 \\
\hline $130-159$ & 12 & 16.2 & 16.2 & 95.9 \\
\hline $160-190$ & 3 & 4.05 & 4.05 & 100.0 \\
\hline Total & 74 & 100.0 & 100.0 & \\
\hline \multicolumn{5}{|c|}{ OR TIME IN HOURS } \\
\hline $2-4$ hours & 3 & 3.8 & 3.8 & 3.8 \\
\hline 4-6 hours & 54 & 67.5 & 67.5 & 71.3 \\
\hline 6-8 hours & 23 & 28.8 & 28.8 & 100.0 \\
\hline Total & 80 & 100.0 & 100.0 & \\
\hline \multicolumn{5}{|c|}{ IONOTROPE DURATION TIME IN HOURS } \\
\hline 2-5 hours & 16 & 20.0 & 20.0 & 20.0 \\
\hline $6-9$ & 17 & 21.3 & 21.3 & 41.3 \\
\hline $10-13$ & 16 & 20.0 & 20.0 & 61.3 \\
\hline $14-17$ & 18 & 22.5 & 22.5 & 83.8 \\
\hline$>18$ hours & 13 & 16.3 & 16.3 & 100.0 \\
\hline Total & 80 & 100.0 & 100.0 & \\
\hline \multicolumn{5}{|c|}{ VENTILATION DURATION } \\
\hline 2-4 hours & 10 & 12.8 & 12.8 & 12.8 \\
\hline 4-6 hours & 21 & 26.9 & 26.9 & 39.7 \\
\hline 6-8 hours & 40 & 51.3 & 51.3 & 91.0 \\
\hline $8-10$ hours & 7 & 9.0 & 9.0 & 100.0 \\
\hline Total & 78 & 100.0 & 100.0 & \\
\hline \multicolumn{5}{|c|}{ REINTUBATION } \\
\hline Yes & 1 & 1.3 & 1.3 & 1.3 \\
\hline No & 77 & 98.7 & 98.7 & 100.0 \\
\hline Total & 78 & 100.0 & & \\
\hline \multicolumn{5}{|c|}{ ICU STAY IN DAYS } \\
\hline $1-2$ days & 53 & 66.3 & 66.3 & 66.3 \\
\hline 2-3 days & 24 & 30.0 & 30.0 & 96.3 \\
\hline 3-4 days & 3 & 3.8 & 3.8 & 100.0 \\
\hline Total & 80 & 100.0 & 100.0 & \\
\hline \multicolumn{5}{|c|}{ POST ICU STAY IN DAYS } \\
\hline 2-3 days & 1 & 1.3 & 1.3 & 1.3 \\
\hline 4-5 days & 9 & 11.3 & 11.3 & 12.5 \\
\hline $6-7$ days & 70 & 87.5 & 87.5 & 100.0 \\
\hline Total & 80 & 100.0 & 100.0 & \\
\hline \multicolumn{5}{|c|}{ HOSPITAL STAY } \\
\hline 4-6 days & 4 & 5.0 & 5.0 & 5.0 \\
\hline 7-9 days & 40 & 50.0 & 50.0 & 55.0 \\
\hline $10-12$ days & 36 & 45.0 & 45.0 & 100.0 \\
\hline Total & 80 & 100.0 & 100.0 & \\
\hline
\end{tabular}




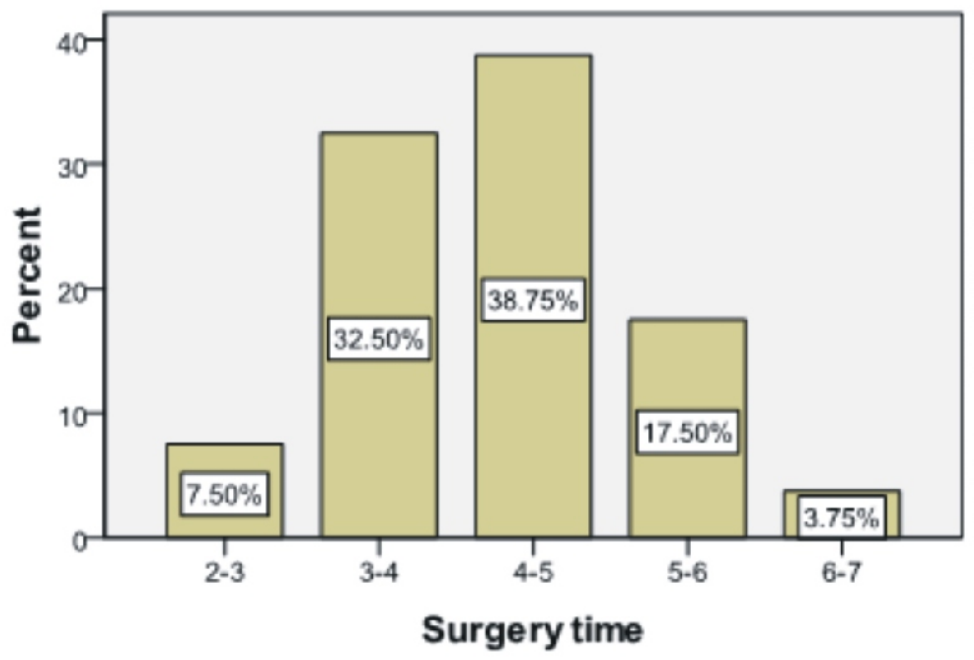

of study by Kataoka G 20 are different than our results. Over all durations in our FTCA study were significantly lower than traditional anaesthesia. In a study conducted by Ender $\mathrm{J}$ 21 Duration of anaesthesia, min (mean + SD) $134.8 \pm 35.2$. In our study only one case was reintubated overall (incidence $1.3 \%$ ) Table 2. A study was conducted by LaheySJetal $^{22}$ showed overall five of 277 (1.8\%) patients had to be re-intubated.

In the present study ICU stay in days was 1-2 days in 53 cases, 2-3 days in 24 cases, 3-4 days in 3 cases. (table2).The table clearly indicate that overwhelming majority (96.3\%) stayed for 1-3 days only. Likewise Post ICU stay was also much shorter 2-3 days 1 case, 4-5 days 9 cases, 6-7 days 70 cases respectively (table2). It is much shorter than CCA as shown by the research of Wong DT 23, which indicated that in CCA approach median post ICU stay was 6 days. In another study 24the result of median stay was 1 hour longer (23 vs $22 \mathrm{~h}, \mathrm{P} \leq 0.001)$. In the present study total hospital stay in days was 4-6 days in 4 case, 7-9 in 40 cases, and 10-12 days in 36 cases (table2). In the study conducted by M, Smith GD (25) the median duration of hospitalization was 6.0 days in both FTCA and CCA group; but the 90th percentile of the hospitalization time was 13 days in the FTCA group and 18 days in the CCA group $(\mathrm{P} \leq$ 0.001). A study conducted by Egger Cheng $\mathrm{DCH}^{26}$ indicated that although all other durations were reduced in FTCA but no significant decrease in hospital Length of stay $(\mathrm{LOS})^{27}$. So for as mortality is concerned, among the 80 cases operated in our center, there was no death; Many studies expressed that If we compare the FTCA with Conventional Cardiac Anaesthesia (CCA) regarding mortality and morbidity, no statistically significant difference in mortality rate and major morbidity was seen ${ }^{28}$, even though there was markedly decreased time to tracheal extubation and decreased ICU LOS in $\mathrm{FTCA}^{29}$. While in other centers, studies do not agree to these results. A metaregression by Van Mastrigt et $a l^{30}$ in 2006 showed that neither anaesthetic dose, nor extubation protocols were significant predictors for myocardial infarction and death following cardiac surgery. However they showed $\neg$ that among 188 patients operated, who were extubated within six hours, one patient died. In another study by Anderson $\mathrm{J}$ 31 however showed a higher mortality ( six of 89, 6.7\%) was seen among patients who required mechanical ventilation for more than six hours after skin closure. In a research conducted by Najafi M. 32 incidence of in-hospital mortality was $1.9 \%$ in the CCA group and $2.3 \%$ in the FTCA group, but difference was not statistically significant ( $\mathrm{p}>.05$ at $95 \% \mathrm{CI}$ ). Compared FTCA with the CCA group, the crude odds ratio for mortality in the FTCA group was 1.20 (95\% confidence interval $0.881 .64, \mathrm{P} 0.25)$ and the adjusted odds ratio was 0.92 (95\% confidence interval, $0.651 .32, \mathrm{P}=$ $0.66)$. Zero mortality in our study can be explained on the basis of small sample size and partially on the expertise of the operating team and after care. Taking into consideration the total scenario we reach to the conclusion that due to known cost benefits, and without compromising mortality, morbidity, there was no reason to continue with high dose opioid techniques. 
Chamchad D, Horrow JC, Nakhamchik L, et $a l^{33}$, showed no difference between early and conventional extubation with regards to mortality in ICU or within 30 days after surgery. There was no significant difference in postoperative myocardial ischemia and re-intubation before or after 24 hours. Early extubation also showed a decreased ICU and hospital length of stay.

Keeping in view the above facts, many cardiac surgical centers have embraced FTCA protocols to reduce ICU bed use and to reduce hospital costs associated with postoperative care. While other researchers ${ }^{34}$ argued that FTCA should not be adopted until further evidence of its safety is available, in particular because the prolonged intensive analgesia resulting from CCA is thought to reduce postoperative myocardial ischemia. Landoni G, Augoustides $^{35}$ have indicated that FTCA is not associated with a higher risk for myocardial ischemia or other perioperative complications compared with conventional anesthetic methods. $^{35}$

\section{CONCLUSION}

Following the intensive discussion, we reach to the conclusion that with the low dosage of anaesthetic medication and reduction for all types of durations under FTCA without any significant difference in mortality and morbidity and with additional significant cost-effective of the cardiac surgeries, the present study shows that FTCA is the genuine need of present time. However this research has certain limitations, particularly its small sample size.

\section{REFERENCES}

1. Pouya Youssefi, David Timbrell etal. Predictors of Failure in Fast Tract Cardiac Surgery..Journal Of Cardiothoracic and Vascular Anesthesia; December 2015 Volume 29, (6)1466-1471.

2. Hantschel D, FasslJ,et al; Fast tract protocol for cardio- anaesthesia. Effective, safe and economical. Aaesthesist. 2009;58:379-386.

3. SalhiyyahK, Elsobky S et al. A clinical and economic evaluatin of Fast Track recovery and cardiac surgery. Heart Surg Forum. 2011;14:e330-E334.

4. Klessling AH,HunekkeP, ReyherC et al.Risk factor analysis for fast track protocol failure.J Cardiothorac Surg. 2013;8:47.

5. Lee A, Zhu F, Underwood M.J. et al, Fast track failure after cardic surgery: External model validation and and implications to ICU bed utilization. Crit Care Med.2013,41:1205-1213.
6. Haanschoten, M.C., van Straten, A.H., ter Woorst, J.F. et al, Fast-track practice in cardiac surgery: results and predictors of outcome. Interact Cardiovasc Thorac Surg. 2012; 15:989-94.

7. Hantschel, D., Fassl, J., Scholz, M. et al, Leipzig fasttrack protocol for cardio-anesthesia. Effective, safe and economical. Anaesthesist. 2009,58:379-386.

8. Zhu F, Lee A, Chee YE. Fast-track cardiac care for adult cardiac surgical patients. Cochrane Database of Systematic Reviews 10: CD003587, 2012.

9. Haanschoten, M.C., van Straten, A.H., ter Woorst, J.F. et al, Fast-track practice in cardiac surgery: results and predictors of outcome. Interact Cardiovasc Thorac Surg. 2012;15:989-94.

10. N.N Hasanein,R.Rashwan etal,Effect of fibro-optic intubation on myocardial ischemia and hormonal stress response on diabetic with ischemic heart disease. Egyptian Journal of Anesthesia2014;26(2):312.

11. Hemmerling TM, Prieto I, Choinière J-L, Basile F, Fortier JD. Ultra-fast-track anesthesia in off-pump coronary artery bypass grafting: A prospective audit comparing opioid-based anesthesia vs thoracic epidural-based anesthesia. Can J Anaesth 2004;51:163-8

12. Cheng DCH: Fast track cardiac surgery pathways: Early extubation, process of care, and cost containment. anesthesiologY 1998; 88:142933

13. Cheng DC: Fast-track cardiac surgery: Economic implications in Post-operative care.J Cardiothorac Vasc Anesth 1998; 12:729

14. Silbert BS, Santamaria JD, O'Brien JL, Blyth CM, Kelly WJ, Molnar RR: The fast track cardiac care team: Early extubation following coronary artery bypass surgery: A prospective randomized controlled trial. Chest 1998; 113:14818

15. London MJ, Shroyer ALW, Grover FL: Fast tracking into the new millennium: An evolving paradigm. anesthesiology 1999; 91:9114

16. Stanley TH, Webster LR: Anesthetic requirements and cardiovascular effects of fentanyloxygen and fentanyldiazepamoxygen anesthesia in man. AnesthAnalg 1978; 57:4116

17. Siliciano D: Con: Early extubation is not preferable to

late extubation in patients undergoing coronary artery surgery. J CardiothoracVascAnesth 1992; 6:4948 
18. Rady MY, Ryan T: Perioperative predictors of extubation failure and the effect on clinical outcome after cardiac surgery. Crit Care Med 1999; 27:3407

19. Michalopoulos A, Nikolaides A, Antzaka C, Deliyanni M, Smirli A, Geroulanos S, Papadimitriou L: Change in anaesthetic practice and postoperative sedation shortens ICU and hospital length of stay following coronary arterybypass surgery. Respir Med 1998; 92:106670

20. Kataoka G, Murai N, Kodera K, Sasaki A, Asano R, Ikeda $\mathrm{M}$, et al. Clinical experience with Smart Care after off-pump coronary artery bypass for early extubation. Journal of Artificial Organs 2007;10(4):218-22

21. Ender J, Borger MA, Scholz M, Funkat AK, Anwar N, Sommer M, et al. Cardiac surgery fast-track treatment in a postanesthetic care unit: six-month results of the Leipzig fast-track concept. Anesthesiology 2008;109:61-6.

22. Lahey SJ, Campos CT, Jennings B, Pawlow P, Stokes T, Levitsky S: Hospital readmission after cardiac surgery: Does 'fast track' cardiac surgery result in costsaving or cost shifting? Circulation 1998; 98:II3540

23. Wong DT, Cheng DCH, Kustra R, Tibshirani R, Karski J, Carroll-Munro J, Sandler AN: Risk factors of delayed extubation, prolonged length of stay in the intensive care unit, and mortality in patients undergoing $\mathrm{CABG}$ with fast track cardiac anesthesia: A new cardiac risk score. anesthesiology 1999; 91:93644

24. Plumer H, Markewitz A, Marhohl K, Bernutz C, Weinhold C: Early extubation after cardiac surgery: A prospective clinical trial including patients at risk. ThoracCardiovascSurg 1998; 46:27580

25. M, Smith GD: Misleading meta-analysis: Lessons learned from 'an effective, safe, simple' intervention that wasn't. BMJ 1995; 310:7524

26. Cheng DCH, Karski J, Peniston C, Asokumar B, Carroll J, Nierenberg H, Roger S, David T, Sandler AN: Morbidity outcome of early versus conventional tracheal extubation following coronary artery bypass graft (CABG) surgery: A prospective randomized, controlled clinical study. J ThoracCardiovascSurg 1996; 112:75564

27. Arom KV, Emery RW, Petersen RJ, Schwartz M: Cost-effectiveness and predictors of early extubation.
Ann ThoracSurg 1995; 60:12732

28. Nicholson DJ, Kowalski SE, Hamilton GA, Meyers MP, Serrette C, Duke PC. Postoperative pulmonary function in coronary artery bypass graft surgery patients undergoing early tracheal extubation: a comparison between short-term mechanical ventilation and early extubation. Journal of Cardiothoracic and Vascular Anesthesia 2002;16(1):27-31.

29. Silbert BS, Santamaria JD, O'Brien JL, Blyth CM, Kelly WJ, Molnar RR, et al. Early extubation following coronary artery bypass surgery: a prospective randomized controlled trial. Chest 1998;113:1481-8

30. vanMastrigt GA, Heijmans J, Severens JL, Fransen EJ, Roekaerts P, Voss G, et al. Short-stay intensive care after coronary artery bypass surgery: randomized clinical trial on safety and cost-effectiveness. Critical Care Medicine 2006;34.(1):65-75

31. Anderson J, Henry L, Hunt S, Ad N. Bispectral index monitoring to facilitate early extubation following cardiovascular surgery. Clinical Nurse Specialist 2010;24(3):140-8.

32. Najafi M. Fast-track method in cardiac surgery: evaluation of risks and benefits of continuous administration technique. Singapore Medical Journal 2008;49(6):470-5. [MEDLINE:

33. Chamchad D, Horrow JC, Nakhamchik L, et al. The impact of immediate extubation in the operating room after cardiac surgery on intensive care and hospital length of stay. Journal of Cardiothoracic and Vascular Anesthesia 2010;24:780-4.

34. Health-related quality of life after fast-track treatment results from a randomized controlled clinical equivalence trial. Quality of Life Research 2010;19(5):631-42.

35. Landoni G, Augoustides JG, Guarracino F, Santini F, Ponschab M, Pasero D, et al. Mortality reduction in cardiac anesthesia and intensive care: results of the first International Consensus Conference. ActaAnaesthesiologicaScandinavica 2011; 55(3): 259-66. [PUBMED: 21288207]

36. Berry P,Thomas S, Mahon S,Jackson M, Fox MA,Fabri B, Weir WI,Russell GN:Myocardial ischaemia after coronary artery bypass grafting:Early vs late extubation. Br J Anaesth 1998;80:20-5 